TRANSACTIONS OF THE

AMERICAN MATHEMATICAL SOCIETY

Volume 357, Number 5 , Pages 2059-2079

S 0002-9947(04)03725-0

Article electronically published on December 9, 2004

\title{
SMALL DEVIATIONS OF WEIGHTED FRACTIONAL PROCESSES AND AVERAGE NON-LINEAR APPROXIMATION
}

\author{
MIKHAIL A. LIFSHITS AND WERNER LINDE
}

\begin{abstract}
We investigate the small deviation problem for weighted fractional Brownian motions in $L_{q}$-norm, $1 \leq q \leq \infty$. Let $B^{H}$ be a fractional Brownian motion with Hurst index $0<H<1$. If $1 / r:=H+1 / q$, then our main result asserts$$
\lim _{\varepsilon \rightarrow 0} \varepsilon^{1 / H} \log \mathbb{P}\left(\left\|\rho B^{H}\right\|_{L_{q}(0, \infty)}<\varepsilon\right)=-c(H, q) \cdot\|\rho\|_{L_{r}(0, \infty)}^{1 / H},
$$

provided the weight function $\rho$ satisfies a condition slightly stronger than the $r$ integrability. Thus we extend earlier results for Brownian motion, i.e. $H=1 / 2$, to the fractional case. Our basic tools are entropy estimates for fractional integration operators, a non-linear approximation technique for Gaussian processes as well as sharp entropy estimates for $l_{q}$-sums of linear operators defined on a Hilbert space.
\end{abstract}

\section{INTRODUCTION}

Let $B^{H}=\left(B^{H}(t)\right)_{t \geq 0}$ be a fractional Brownian motion with Hurst index $H \in$ $(0,1)$, i.e. $B^{H}$ is a centered Gaussian process with a.s. continuous paths and covariance

$$
\mathbb{E} B^{H}(t) B^{H}\left(t^{\prime}\right)=\frac{1}{2}\left\{|t|^{2 H}+\left|t^{\prime}\right|^{2 H}-\left|t-t^{\prime}\right|^{2 H}\right\}, \quad t, t^{\prime} \geq 0 .
$$

Given a measurable weight function $\rho:(0, \infty) \rightarrow[0, \infty)$ such that we have for some $q \in[1, \infty]$

$$
\mathbb{P}\left(\left\|\rho B^{H}\right\|_{L_{q}(0, \infty)}<\infty\right)=1
$$

we are mainly interested in the behaviour of

$$
\log \mathbb{P}\left(\left\|\rho B^{H}\right\|_{L_{q}(0, \infty)}<\varepsilon\right)
$$

as $\varepsilon \rightarrow 0$. For $\rho=\mathbf{1}_{[0,1]}$ this question was investigated in [24], [19], 11] and [17]. The final result is as follows: For $1 \leq q \leq \infty$ the limit

$$
\lim _{\varepsilon \rightarrow 0} \varepsilon^{1 / H} \log \mathbb{P}\left(\left\|B^{H}\right\|_{L_{q}[0,1]}<\varepsilon\right):=-c(H, q)
$$

exists with finite $c(H, q)>0$.

Received by the editors May 27, 2003 and, in revised form, December 18, 2003.

2000 Mathematics Subject Classification. Primary 60G15; Secondary 47B06, 47B10, 47G10.

Key words and phrases. Fractional Brownian motion, small deviations, fractional integration operator, entropy numbers, non-linear approximation.

The authors were supported in part by DFG-RFBR Grant 99-01-04027 and by RFBR Grant 02-01-00265.

(C)2004 American Mathematical Society Reverts to public domain 28 years from publication 
If $H=1 / 2$, then $B^{H}$ is a standard Wiener process $W$. In this case the behaviour of (1.2) was thoroughly investigated in [16]. To formulate the main result of this paper, we need the following notion: For $q \in[1, \infty]$ and $0<H<1$ define the number $r>0$ by

$$
\frac{1}{r}:=H+\frac{1}{q}
$$

Using this $r>0$ we construct a new norm for functions $\rho$ on $(0, \infty)$ by

$$
|\rho|_{r}:=\left(\sum_{k=-\infty}^{\infty} 2^{k r H}\|\rho\|_{L_{q}\left[2^{k}, 2^{k+1}\right]}^{r}\right)^{1 / r} .
$$

The main result in [16] asserts for $H=1 / 2$, i.e. for Brownian motion,

$$
\lim _{\varepsilon \rightarrow 0} \varepsilon^{2} \log \mathbb{P}\left(\|\rho W\|_{L_{q}(0, \infty)}<\varepsilon\right)=-c(1 / 2, q) \cdot\|\rho\|_{L_{r}(0, \infty)}^{2}
$$

provided $|\rho|_{r}<\infty$. Observe that Hölder's inequality yields

$$
\|\rho\|_{L_{r}(0, \infty)}:=\|\rho\|_{r} \leq|\rho|_{r}
$$

yet it is not difficult to construct $\rho$ 's with $\|\rho\|_{r}<\infty$ and $|\rho|_{r}=\infty$.

The main result of the present paper is the extension of (1.6) to all $H \in(0,1)$, i.e. if $|\rho|_{r}<\infty$, then we have

$$
\lim _{\varepsilon \rightarrow 0} \varepsilon^{1 / H} \log \mathbb{P}\left(\left\|\rho B^{H}\right\|_{L_{q}(0, \infty)}<\varepsilon\right)=-c(H, q) \cdot\|\rho\|_{L_{r}(0, \infty)}^{1 / H}
$$

with $c(H, q)$ and $r$ defined by (1.3) and (1.4), respectively.

When treating small deviation problems for $\rho B^{H}$ with $H \neq 1 / 2$, completely new methods are required. These methods are of analytical nature. It is well known that fractional Brownian motions are tightly related to fractional integration operators of Riemann-Liouville type (cf. [18]). Thus, by the results in [8] and [12 the small deviation problem for weighted fractional motions turns out to be equivalent to the study of the asymptotic behavior of the entropy numbers for certain weighted fractional integration operators.

It is convenient to solve this analytical problem in a more general context, namely to investigate the degree of compactness of $l_{q}$-sums of operators. More precisely, let $\mathcal{H}$ be a Hilbert space and let $T_{j}, j=1,2, \ldots$, be operators from $\mathcal{H}$ into some Banach spaces $E_{j}$. If

$$
E^{q}:=l_{q}\left(E_{j}: j \in \mathbb{N}\right)=\left\{x=\left(x_{j}\right)_{j \geq 1}: x_{j} \in E_{j},\left\|\left(\left\|x_{j}\right\|\right)\right\|_{l_{q}}<\infty\right\},
$$

then (under some obvious assumptions) the family $\left(T_{j}\right)_{j \geq 1}$ generates in a canonical way an operator $T^{q}$ from $\mathcal{H}$ into $E^{q}$. The main result in the functional analytic part of this paper is an estimate for the entropy numbers $e_{n}\left(T^{q}\right)$ by means of $e_{n}\left(T_{j}\right), j \in \mathbb{N}$. Surprisingly, the proof of this requires completely different methods for the two cases $q<\infty$ and $q=\infty$, respectively. For $q<\infty$ so-called average Kolmogorov numbers play a central role. They describe how good a non-linear approximation of a Gaussian vector by finite rank vectors can be. These numbers were introduced and investigated in the theory of information-based complexity; their basic properties can be found in [6].

In the case $q=\infty$ the technique of average Kolmogorov numbers does not work. Instead we use strong results due to B. Carl about compactness properties of Hölder 
operators. In our construction, the essential and (as far as we know) new idea is to investigate bundles of metric spaces and their metric entropy.

It is remarkable that we use analytic methods for proving probabilistic bounds while probabilistic tools work for proving analytic estimates. Let us note, however, that a part of our analytic arguments can be expressed directly in probabilistic language; see 7]. But we believe that our analytic methods are more general and could therefore also be applied for the investigation of other classes of fractional processes.

The paper is organized as follows. Section 2 provides a minimal necessary analytical background as e.g. the basic estimates of entropy numbers for $l_{q}$-sums of operators. Weighted fractional integration operators are treated in Section 3. The small deviation results are stated and proved in Section 4. Finally, the detailed exposition of the analytic approach is presented in Sections 5 and 6 for finite and infinite $q$, respectively. These last two sections are not necessary for those who are only interested in probabilistic applications. On the other hand, "pure analysts" may skip Section 4 and read Sections 5 and 6 directly.

\section{ENTROPY NUMBERS OF $l_{q}$-SUMS OF OPERATORS}

For any sequence of Banach spaces $\left(E_{j}\right)_{j \geq 1}$ and a number $q \in[1, \infty)$, we define the $l_{q}$-sum $E^{q}$ by

$$
E^{q}:=l_{q}\left(E_{j}: j \in \mathbb{N}\right)=\left\{x=\left(x_{j}\right)_{j \geq 1}: x_{j} \in E_{j}, \sum_{j=1}^{\infty}\left\|x_{j}\right\|^{q}<\infty\right\}
$$

and endow it with the norm

$$
\|x\|_{q}:=\left(\sum_{j=1}^{\infty}\left\|x_{j}\right\|^{q}\right)^{1 / q}, \quad x=\left(x_{j}\right)_{j \geq 1} .
$$

If linear operators $T_{j}$ from a Hilbert space $\mathcal{H}$ into $E_{j}$ satisfy

$$
\sup _{\|h\| \leq 1} \sum_{j=1}^{\infty}\left\|T_{j} h\right\|^{q}<\infty
$$

then the operator $T^{q}: \mathcal{H} \rightarrow E^{q}$ defined by $T^{q} h:=\left(T_{j} h\right)_{j \geq 1}, h \in \mathcal{H}$, is bounded. We call $T^{q}$ the $l_{q}$-sum of the $T_{j}$ 's. Assume now that each operator $T_{j}$ has a certain degree of compactness. Then it is natural to investigate the degree of compactness of $T^{q}$. To be more precise, recall the notion of entropy numbers, a suitable measure of compactness. Let $T: \mathcal{H} \rightarrow E$ be a linear operator. Its $n$-th (dyadic) entropy number is then defined by

$$
e_{n}(T):=\inf \left\{\varepsilon>0: T\left(U_{\mathcal{H}}\right) \subseteq \bigcup_{j=1}^{2^{n-1}}\left\{T h_{j}+\varepsilon U_{E}\right\}, h_{j} \in U_{\mathcal{H}}\right\},
$$

where $U_{\mathcal{H}}$ and $U_{E}$ are the unit balls in $\mathcal{H}$ and $E$, respectively. The previous question can now be formulated as follows. Suppose that

$$
\sup _{n \geq 1} n^{1 / \gamma} e_{n}\left(T_{j}\right):=\kappa_{j}<\infty
$$

for a certain $\gamma>0$ and all $j \in \mathbb{N}$. What can be said about $\sup _{n \geq 1} n^{1 / \gamma} e_{n}\left(T^{q}\right)$ ?

The main objective of Section $[5$ is to provide the following answer. 
Theorem 2.1. For $1 \leq j<\infty$ let $T_{j}$ be operators from a separable Hilbert space $\mathcal{H}$ into Banach spaces $E_{j}$. Suppose that for some $\gamma<2$ we have (2.1). Then, if $1 \leq q<\infty$, this implies

$$
\sup _{n \geq 1} n^{1 / \gamma} e_{n}\left(T^{q}\right) \leq c \cdot\left(\sum_{j=1}^{\infty} \kappa_{j}^{r}\right)^{1 / r}
$$

with $1 / r=1 / \gamma+1 / q-1 / 2$ and $c>0$ depending only on $q$ and $\gamma$.

The notions of $E^{q}$ and $T^{q}$ make sense for $q=\infty$ as well. Yet, unfortunately, the proof of Theorem 2.1 cannot be extended to this case. Thus the following question remains open.

Problem. Let $\gamma<2$ and $1 / r:=1 / \gamma-1 / 2$. Suppose that operators $T_{j}: \mathcal{H} \rightarrow E_{j}$ satisfy (2.1). Does this imply for $T^{\infty}: \mathcal{H} \rightarrow E^{\infty}$ that

$$
\sup _{n \geq 1} n^{1 / \gamma} e_{n}\left(T^{\infty}\right) \leq c \cdot\left(\sum_{j=1}^{\infty} \kappa_{j}^{r}\right)^{1 / r} ?
$$

Our next aim is to formulate a result (proved in Section 6 below) asserting that (2.3) is valid under certain stronger assumptions than (2.1), namely, under certain additional properties of the $E_{j}$ 's as well as of the type of continuity of the $T_{j}$ 's. We start with specifying the image spaces $E_{j}$. Let $\left(K_{j}, d_{j}\right), j \in \mathbb{N}$, be compact metric spaces. Then $C\left(K_{j}, d_{j}\right)$ denote the Banach spaces of continuous functions on $K_{j}$. This time compactness properties of the $K_{j}$ 's are expressed in terms of (non-dyadic) entropy numbers. If $(K, d)$ is a metric space, then its entropy numbers are defined by

$$
\varepsilon_{n}(K, d):=\inf \left\{\varepsilon>0: \exists A_{1}, \ldots, A_{n}: K=\bigcup_{i=1}^{n} A_{i}, \operatorname{diam}\left(A_{i}\right) \leq \varepsilon\right\} .
$$

Next we have to specify the properties of the treated operators. Let $T$ be an operator from $\mathcal{H}$ with values in $C(K, d)$ for some compact metric space $K$. Given $\alpha \in(0,1]$, it is said to be an $\alpha$-Hölder operator (with $\alpha$-Hölder norm $\|T\|_{\alpha}$ ) provided that

$$
\|T\|_{\alpha}:=\sup _{\|h\| \leq 1} \sup _{t \neq t^{\prime}} \frac{\left|(T h)(t)-(T h)\left(t^{\prime}\right)\right|}{d\left(t, t^{\prime}\right)^{\alpha}}<\infty .
$$

Now we are in a position to state the substitute for Theorem 2.1 in the case $q=\infty$.

Theorem 2.2. Let $T_{j}: \mathcal{H} \rightarrow C\left(K_{j}, d_{j}\right)$ be $\alpha$-Hölder operators for some $\alpha \in(0,1]$ and suppose that for each $j, n \in \mathbb{N}$ we have $\varepsilon_{n}\left(K_{j}, d_{j}\right) \leq \rho_{j} \cdot n^{-1 / \sigma}$ for certain $\rho_{j}>0$ and some $\sigma>0$. If

$$
T^{\infty}: \mathcal{H} \rightarrow l_{\infty}\left(C\left(K_{j}, d_{j}\right): j \in \mathbb{N}\right)
$$

is defined by $T^{\infty} h:=\left(T_{j} h\right)_{j \geq 1}$, then

$$
e_{n}\left(T^{\infty}\right) \leq c \cdot \tilde{\rho} \cdot n^{-1 / 2-\alpha / \sigma}
$$

where $\tilde{\rho}:=\left(\sum_{j=1}^{\infty}\left\|T_{j}\right\|_{\alpha}^{\sigma / \alpha} \rho_{j}^{\sigma}\right)^{\alpha / \sigma}$. 
Remark 2.3. Let us compare Theorem 2.2 with Theorem 2.1 If the $T_{j}$ 's from $\mathcal{H}$ into $C\left(K_{j}, d_{j}\right)$ are 1-Hölder with $\left\|T_{j}\right\|_{1} \leq 1$ (note that this can always be achieved by changing the metrics suitably) and if the compact spaces $K_{j}$ satisfy for some $\gamma<2$ the assumption

$$
\varepsilon_{n}\left(K_{j}, d_{j}\right) \leq \kappa_{j} \cdot n^{-1 / \gamma+1 / 2},
$$

then we derive on one hand (see Theorem 6.2 below)

$$
e_{n}\left(T_{j}\right) \leq c \cdot \kappa_{j} \cdot n^{-1 / \gamma}
$$

and on the other hand (by Theorem 2.2)

$$
e_{n}\left(T^{\infty}\right) \leq c \cdot\left(\sum_{j=1}^{\infty} \kappa_{j}^{r}\right)^{1 / r} \cdot n^{-1 / \gamma},
$$

where $1 / r=1 / \gamma-1 / 2$. For $q<\infty$, Theorem 2.1 allows us to derive (2.6) (with adequate $r$ ) directly from (2.5) while for $q=\infty$ we need (2.4) together with the Hölder condition for the $T_{j}$ 's.

\section{Degree of COMPACTNESS OF FRACTIONAL INTEGRATION OPERATORS}

For $H \in(0,1)$ we shall investigate the following two integral operators:

$$
\begin{aligned}
\left(R_{H} f\right)(t) & :=\int_{0}^{t}(t-s)^{H-1 / 2} f(s) d s, \quad t \geq 0, \\
\left(B_{H} f\right)(t) & :=\int_{-\infty}^{t}\left[(t-s)^{H-1 / 2}-(-s)_{+}^{H-1 / 2}\right] f(s) d s, \quad t \geq 0 .
\end{aligned}
$$

As usual we set $(x)_{+}=\max \{x, 0\}$ for $x \in \mathbb{R}$. It is well known that for any bounded interval $I \subseteq[0, \infty)$ and for any $p \in[1, \infty]$ both operators are bounded as mappings from $L_{2}(\mathbb{R})$ into $L_{p}(I)$. The operator $R_{H}$ is usually called (up to some factor) a fractional integration operator of Riemann-Liouville type and $B_{H}$ appears in a natural way in the theory of fractional Brownian motion.

For later purposes it is also important to investigate their difference $Q_{H}:=$ $B_{H}-R_{H}$ acting as follows:

$$
\left(Q_{H} f\right)(t):=\int_{-\infty}^{0}\left[(t-s)^{H-1 / 2}-(-s)^{H-1 / 2}\right] f(s) d s, \quad t \geq 0 .
$$

We need the following two known properties of these operators:

(1) For $1 \leq p \leq \infty$ we have (cf. [12] or [2])

$$
e_{n}\left(R_{H}: L_{2}(\mathbb{R}) \rightarrow L_{p}[0,1]\right) \approx n^{-H-1 / 2} .
$$

(2) For each $H \in(0,1)$ there exist constants $c_{1}, c_{2}>0$ such that (cf. [2])

$$
e_{n}\left(Q_{H}: L_{2}(\mathbb{R}) \rightarrow L_{p}[0,1]\right) \leq c_{1} e^{-c_{2} n^{1 / 3}} .
$$

Consequently, in view of (3.4) and (3.5) we also have

$$
e_{n}\left(B_{H}: L_{2}(\mathbb{R}) \rightarrow L_{p}[0,1]\right) \approx n^{-H-1 / 2} .
$$

Now let $I$ be a bounded interval in $[0, \infty)$ and regard $B_{H}$ as an operator from $L_{2}(\mathbb{R})$ into $L_{p}(I)$. Then, of course, the asymptotic behaviour of $e_{n}\left(B_{H}: L_{2}(\mathbb{R}) \rightarrow L_{p}(I)\right)$ is of order $n^{-H-1 / 2}$ as well. For our later purposes it is important to know how the asymptotic depends on size and position of the interval $I$. The next theorem answers this question. 
Theorem 3.1. Let $I=\left[s_{1}, s_{2}\right]$ be a bounded interval in $[0, \infty)$. Regard $B_{H}$ as an operator from $L_{2}(\mathbb{R})$ into $L_{p}(I)$ for some $p \in[1, \infty]$. Then there are operators $S_{H}$ and $F_{H}$ from $L_{2}(\mathbb{R})$ into $L_{p}(I)$ possessing the following properties:

(a) $B_{H}=S_{H}+F_{H}$,

(b) $\quad e_{n}\left(S_{H}\right) \leq c \cdot|I|^{H+1 / p} \cdot n^{-H-1 / 2}$,

(c) $\quad \operatorname{rank}\left(F_{H}\right)=1$ and $\left\|F_{H}\right\| \leq C \cdot s_{1}^{H} \cdot|I|^{1 / p}$.

Proof. Defining $F_{H}$ by

$$
\left(F_{H} f\right)(t)=\int_{-\infty}^{s_{1}}\left[\left(s_{1}-s\right)^{H-1 / 2}-(-s)_{+}^{H-1 / 2}\right] f(s) d s, \quad t \in I,
$$

the properties in (c) may be proved easily with

$$
C^{2}=\int_{-\infty}^{1}\left[(1-s)^{H-1 / 2}-(-s)_{+}^{H-1 / 2}\right]^{2} d s .
$$

If $S_{H}: L_{2}(\mathbb{R}) \rightarrow L_{p}(I)$ is defined by

$$
\left(S_{H} f\right)(t):=\int_{-\infty}^{t}\left[(t-s)^{H-1 / 2}-\left(s_{1}-s\right)_{+}^{H-1 / 2}\right] f(s) d s, \quad t \in I,
$$

then decomposition (a) holds, and a simple scaling transformation gives

$$
e_{n}\left(S_{H}\right)=|I|^{H+1 / p} \cdot e_{n}\left(B_{H}: L_{2} \rightarrow L_{p}[0,1]\right) .
$$

Thus in view of (3.6) it follows that $e_{n}\left(S_{H}\right) \leq c \cdot|I|^{H+1 / p} \cdot n^{-H-1 / 2}$.

Let $\rho:(0, \infty) \rightarrow[0, \infty)$ be an $L_{q}$-weight function, i.e. it is measurable and satisfies $\rho \cdot \mathbf{1}_{[x, y]} \in L_{q}$ for any real numbers $0<x<y$. Consequently, $\rho B_{H}$ is well defined as mapping from $L_{2}(\mathbb{R})$ into $L_{q}(0, \infty)$ and our aim is to find suitable estimates for its entropy numbers (in dependence of $\rho$ ).

Theorem 3.2. For $0<H<1,1 \leq q \leq \infty$, let $\rho$ be as above and regard $\rho B_{H}$ as mapping from $L_{2}(\mathbb{R})$ into $L_{q}(0, \infty)$. Then

$$
\sup _{n \geq 1} n^{H+1 / 2} e_{n}\left(\rho B_{H}\right) \leq c \cdot|\rho|_{r}
$$

with $r$ and $|\rho|_{r}$ defined by (1.4) and (1.5), respectively.

Proof. For the dyadic intervals $\Delta_{k}:=\left[2^{k}, 2^{k+1}\right], k \in \mathbb{Z}$, we define operators $B_{H}^{k}$, $k \in \mathbb{Z}$, by

$$
\left(B_{H}^{k} f\right)(t)=\int_{-\infty}^{t}\left[(t-s)^{H-1 / 2}-(-s)_{+}^{H-1 / 2}\right] f(s) d s, \quad t \in \Delta_{k} .
$$

If $f \in L_{2}(\mathbb{R})$, then we have

$$
\left\|\rho B_{H} f\right\|_{q}=\left\|\sum_{k=-\infty}^{\infty}\left(\rho \cdot \mathbf{1}_{\Delta_{k}}\right)\left(B_{H}^{k} f\right)\right\|_{q} \leq\left(\sum_{k=-\infty}^{\infty}\|\rho\|_{L_{q}\left(\Delta_{k}\right)}^{q} \cdot\left\|B_{H}^{k} f\right\|_{\infty}^{q}\right)^{1 / q} .
$$

Setting

$$
\rho_{k}:=\|\rho\|_{L_{q}\left(\Delta_{k}\right)},
$$

the operator

$$
B_{H, \rho}^{\infty}: L_{2}(\mathbb{R}) \rightarrow l_{q}\left(C\left(\Delta_{k}\right): k \in \mathbb{Z}\right)
$$


is defined by $B_{H, \rho}^{\infty} f:=\left(\rho_{k} \cdot B_{H}^{k} f\right)_{k \in \mathbb{Z}^{*}}$. Estimate (3.11) may now be rewritten as

$$
\left\|\rho B_{H} f\right\|_{q} \leq\left\|B_{H, \rho}^{\infty} f\right\|_{l_{q}\left(C\left(\Delta_{k}\right)\right)}
$$

for $f \in L_{2}(\mathbb{R})$. This is the place where the $l_{q}$-sums from Section 2 come into consideration. It is easy to see (cf. [16], Lemma 4.2) that (3.13) implies

$$
e_{n}\left(\rho B_{H}\right) \leq e_{n}\left(B_{H, \rho}^{\infty}\right),
$$

thus it remains to estimate the right-hand side of (3.14). This is done for $q<\infty$ and $q=\infty$ by different methods.

Let us start with $1 \leq q<\infty$. By Theorem 3.1 the operator $\rho_{k} B_{H}^{k}$ (with $\rho_{k}$ defined by (3.12) and $B_{H}^{k}$ by (3.10) ) can be written as

$$
\rho_{k} B_{H}^{k}=\rho_{k} S_{H}^{k}+\rho_{k} F_{H}^{k}
$$

where $S_{H}^{k}, F_{H}^{k}: L_{2}(\mathbb{R}) \rightarrow L_{\infty}\left(\Delta_{k}\right)$ possess the properties stated in Theorem 3.1 for $p=\infty$. In particular, assertion (b) of Theorem 3.1yields

$$
\sup _{n \geq 1} n^{1 / 2+H} e_{n}\left(\rho_{k} S_{H}^{k}\right) \leq c \cdot \rho_{k} \cdot 2^{k H}
$$

while its assertion (c) leads to (use 1.3.36 in [5])

$$
\begin{aligned}
\sup _{n \geq 1} n^{1 / 2+H} e_{n}\left(\rho_{k} F_{H}^{k}\right) & \leq c \cdot \rho_{k} \cdot \sup _{n \geq 1} 2^{n-1} e_{n}\left(F_{H}^{k}\right) \\
& \leq c \cdot \rho_{k} \cdot\left\|F_{H}^{k}\right\| \leq c \cdot \rho_{k} \cdot 2^{k H} .
\end{aligned}
$$

We now apply Theorem 2.1 with $1 / \gamma=H+1 / 2$, i.e. it holds that

$$
1 / r=1 / \gamma+1 / q-1 / 2=H+1 / q,
$$

hence (3.16) leads to

$$
\sup _{n \geq 1} n^{1 / 2+H} e_{n}\left(\left(\rho_{k} S_{H}^{k}\right)_{k \in \mathbb{Z}}\right) \leq c \cdot\left(\sum_{k=-\infty}^{\infty} \rho_{k}^{r} 2^{k H r}\right)^{1 / r}=c \cdot|\rho|_{r} .
$$

By similar reason (3.17) implies

$$
\sup _{n \geq 1} n^{1 / 2+H} e_{n}\left(\left(\rho_{k} F_{H}^{k}\right)_{k \in \mathbb{Z}}\right) \leq c \cdot|\rho|_{r}
$$

and since (3.15) yields $B_{H, \rho}^{\infty}=\left(\rho_{k} S_{H}^{k}\right)_{k \in \mathbb{Z}}+\left(\rho_{k} F_{H}^{k}\right)_{k \in \mathbb{Z}}$ we derive (3.9) from (3.14), (3.18) and (3.19). This completes the proof in the case $q<\infty$.

Assume now $q=\infty$. Our aim is to apply Theorem 2.2. Therefore, we have to evaluate the Hölder continuity of $B_{H}^{k}$ as well as the degree of compactness of $\Delta_{k}$. By trivial calculations $\rho_{k} B_{H}^{k}$ is $H$-Hölder with $H$-Hölder norm

$$
\left\|\rho_{k} B_{H}^{k}\right\|_{H}=C \cdot \rho_{k}, \quad k \in \mathbb{Z}
$$

with $C$ defined in (3.7). On the other hand, of course, it holds that

$$
\varepsilon_{n}\left(\Delta_{k},|\cdot|\right) \approx\left|\Delta_{k}\right| \cdot n^{-1} \text {. }
$$

Consequently, an application of Theorem 2.2 (with $\sigma=1$ and $\alpha=H$ ) leads by (3.20) and (3.21) to

$$
\sup _{n \geq 1} n^{1 / 2+H} e_{n}\left(B_{H, \rho}^{\infty}\right) \leq c \cdot\left(\sum_{k=-\infty}^{\infty} \rho_{k}^{1 / H}\left|\Delta_{k}\right|\right)^{H}=c \cdot|\rho|_{r} .
$$

Note that $1 / r=H$ in this case. Finally (3.14) completes the proof for $q=\infty$. 


\section{Small Deviations of Weighted FRACtional PROCESSES}

Let $W$ be a Wiener process on $[0, \infty)$. Then, if $0<H<1$, we consider the following two fundamental fractional processes:

$$
\begin{aligned}
R^{H}(t) & :=\int_{0}^{t}(t-s)^{H-1 / 2} d W(s), \quad t \geq 0, \\
B^{H}(t) & :=\int_{-\infty}^{t}\left[(t-s)^{H-1 / 2}-(-s)_{+}^{H-1 / 2}\right] d W(s), \quad t \geq 0 .
\end{aligned}
$$

The process $\left(R^{H}(t)\right)_{t \geq 0}$ is usually called the Riemann-Liouville process of order $H$. It makes sense for all $H>0$, yet since we are mainly interested in the relation between $R^{H}$ and $B^{H}$ we restrict ourselves to $H<1$. The process $\left(B^{H}(t)\right)_{t \geq 0}$ is up to some factor the classical fractional Brownian motion as introduced in (1.1) (with Hurst index $H$ ). Of course, for $H=1 / 2$ both processes coincide with $W$.

The processes $R^{H}$ and $B^{H}$ are tightly related with the operators $R_{H}$ and $B_{H}$ introduced in (3.1) and (3.2), respectively. Indeed, if $\left(f_{k}\right)_{k \geq 1}$ is any ONB in $L_{2}(\mathbb{R})$ and if the $\xi_{k}$ 's are $\mathcal{N}(0,1)$-distributed i.i.d. variables, then the random functions

$$
\begin{aligned}
& t \rightarrow \sum_{k=1}^{\infty} \xi_{k}\left(R_{H} f_{k}\right)(t), \quad t \geq 0, \quad \text { and } \\
& t \rightarrow \sum_{k=1}^{\infty} \xi_{k}\left(B_{H} f_{k}\right)(t), \quad t \geq 0,
\end{aligned}
$$

are Riemann-Liouville and fractional Brownian motion processes of order $H$, respectively.

It is known (cf. [1] and [17]) that for any $q \in[1, \infty]$ there exist the finite positive small ball limits

$$
\begin{aligned}
c(H, q) & :=-\lim _{\varepsilon \rightarrow 0} \varepsilon^{1 / H} \log \mathbb{P}\left(\left\|R^{H}\right\|_{L_{q}[0,1]} \leq \varepsilon\right) \\
& =-\lim _{\varepsilon \rightarrow 0} \varepsilon^{1 / H} \log \mathbb{P}\left(\left\|B^{H}\right\|_{L_{q}[0,1]} \leq \varepsilon\right) .
\end{aligned}
$$

Suppose now we are given a (measurable) weight function $\rho:(0, \infty) \rightarrow[0, \infty)$ such that for some $q \in[1, \infty]$ we have $\mathbb{P}\left(\left\|\rho B^{H}\right\|_{L_{q}(0, \infty)}<\infty\right)=1$. In this case one may ask whether or not $\lim _{\varepsilon \rightarrow 0} \varepsilon^{1 / H} \log \mathbb{P}\left(\left\|\rho B^{H}\right\|_{L_{q}(0, \infty)} \leq \varepsilon\right)$ exists as well and if so, how it depends on $\rho$. Similar questions may be posed for $R^{H}$.

A first step toward an answer to the above questions is given by the following estimates.

Theorem 4.1. For $q \in[1, \infty]$ let $r>0$ be defined by 1.4. Then

$$
\begin{aligned}
& \liminf _{\varepsilon \rightarrow 0} \varepsilon^{1 / H} \log \mathbb{P}\left(\left\|\rho R^{H}(t)\right\|_{q} \leq \varepsilon\right) \\
& \quad \geq \liminf _{\varepsilon \rightarrow 0} \varepsilon^{1 / H} \log \mathbb{P}\left(\left\|\rho B^{H}(t)\right\|_{q} \leq \varepsilon\right) \geq-c \cdot|\rho|_{r}^{1 / H}
\end{aligned}
$$

for some $c>0$ depending only on $q$ and $H$.

Proof. The first inequality is a direct consequence of Anderson's inequality (see [1] or [14], Chapter 11). The second one immediately follows from Theorem [3.2] and the results in [8] and [12] linking small deviations and entropy numbers. 
Metric entropy tools are too crude for getting sharp small deviation estimates as stated in (4.2) or (4.3). For this purpose, more precise probabilistic methods are necessary. Nevertheless, Theorem 4.1 will play an important role in eliminating some disturbing remainder term in Theorem 4.6 below.

Before proceeding further, we state two lemmas for later use. The first one is a partial solution of the so-called correlation conjecture. It was first proved in the necessary generality in [9] as an extension of [23]; see also Theorem 2.14 in [13]. We formulate it here in the form appropriate for our own use.

Lemma 4.2. For every $\eta \in(0,1)$ there exists a constant $K=K(\eta)>0$ such that for every $u>0$ and every pair of centered jointly Gaussian random vectors $X_{1}, X_{2}$ with values in a Banach space $E$ one has

$$
\mathbb{P}\left(\left\|X_{1}+X_{2}\right\| \leq u\right) \geq \mathbb{P}\left(\left\|X_{1}\right\| \leq(1-\eta) u\right) \mathbb{P}\left(\left\|X_{2}\right\| \leq u / K\right) .
$$

The next result is a direct consequence of properties of $Q_{H}$ defined in (3.3).

Lemma 4.3. Let $I \subseteq[0, \infty)$ be a finite interval and let $\left(I_{j}\right)_{j=1}^{m}$ be a finite partition of $I$ into intervals $I_{j}=\left[s_{j}, s_{j+1}\right]$ with $s_{1}<\ldots<s_{m+1}$. Define the stochastic process $Z^{H}=\left(Z^{H}(t)\right)_{t \in I}$ by

$$
Z^{H}(t):=\int_{-\infty}^{s_{j}}\left[(t-s)^{H-1 / 2}-(-s)_{+}^{H-1 / 2}\right] d W(s), \quad t \in I_{j} .
$$

If $\rho=\sum_{j=1}^{m} \rho_{j} \mathbf{1}_{I_{j}}$ for some $\rho_{j} \geq 0$, then for each $\gamma>0$ and $q \in[1, \infty]$ there is a constant $c>0$ (depending on $q, \gamma, \rho$ and $I$ ) such that

$$
\log \mathbb{P}\left(\left\|\rho Z^{H}\right\|_{L_{q}(I)} \leq \varepsilon\right) \geq-c \cdot \varepsilon^{-\gamma}, \quad \varepsilon>0 .
$$

Proof. Define an operator $T_{H}^{j}: L_{2}(\mathbb{R}) \rightarrow L_{q}\left(I_{j}\right)$ by

$$
\begin{aligned}
\left(T_{H}^{j} f\right)(t) & :=\int_{-\infty}^{s_{j}}\left[(t-s)^{H-1 / 2}-(-s)_{+}^{H-1 / 2}\right] f(s) d s \\
& =\int_{-\infty}^{s_{j}}\left[(t-s)^{H-1 / 2}-\left(s_{j}-s\right)^{H-1 / 2}\right] f(s) d s \\
& +\int_{-\infty}^{s_{j}}\left[\left(s_{j}-s\right)^{H-1 / 2}-(-s)_{+}^{H-1 / 2}\right] f(s) d s
\end{aligned}
$$

where the first operator is equivalent to $\left|I_{j}\right|^{H+1 / q} Q_{H}$ while the second one has rank one. Thus by (3.5) and estimate 1.3.36 in [5] it follows that

$$
e_{n}\left(\rho_{j} T_{H}^{j}\right) \leq c_{1} \cdot e^{-c_{2} n^{1 / 3}}
$$

for certain $c_{1}, c_{2}>0$ depending on $\rho_{j}, q, H$ and $I_{j}$. Of course, by the additivity of entropy numbers estimate (4.6) remains valid (with different constants) for $e_{n}\left(\sum_{j=1}^{m} \rho_{j} T_{H}^{j}\right)$. In particular, this implies

$$
e_{n}\left(\sum_{j=1}^{m} \rho_{j} T_{H}^{j}\right) \leq c \cdot n^{-1 / \theta}
$$

for any $\theta<2$. Finally, by Theorem 5.1 in [12] we derive from (4.7)

$$
\log \mathbb{P}\left(\left\|\rho Z^{H}\right\|_{L_{q}(I)} \leq \varepsilon\right) \geq-c^{\prime} \cdot \varepsilon^{-2 \theta /(2-\theta)},
$$

completing the proof. 
Our next aim is to precise the lower bound in (4.4) for $\rho$ 's possessing bounded support.

Theorem 4.4. Let $I \subseteq[0, \infty)$ be a bounded interval and suppose $\rho \in L_{q}(I)$ for some $q \in[1, \infty)$. Then

$$
\liminf _{\varepsilon \rightarrow 0} \varepsilon^{1 / H} \log \mathbb{P}\left(\left\|\rho B^{H}\right\|_{L_{q}(I)} \leq \varepsilon\right) \geq-c(H, q) \cdot\|\rho\|_{L_{r}(I)}^{1 / H},
$$

where $r$ and $c(H, q)$ are as in (1.4) and (1.5), respectively.

Proof. In a first step we prove (4.8) for interval step functions $\rho$, i.e. we assume

$$
\rho=\sum_{j=1}^{m} \rho_{j} \mathbf{1}_{I_{j}}
$$

for certain $\rho_{j} \geq 0$ and intervals $I_{j}=\left[s_{j}, s_{j+1}\right]$ with $s_{1}<\ldots<s_{m+1}$ and $I=$ $\left[s_{1}, s_{m+1}\right]$. Consequently, for such a function $\rho$ we may represent $\rho B^{H}$ as

$$
\rho B^{H}=\rho Z^{H}+\sum_{j=1}^{m} \rho_{j} R_{j}^{H}
$$

with $Z^{H}$ defined in (4.5) and

$$
R_{j}^{H}(t):=\int_{s_{j}}^{t}(t-s)^{H-1 / 2} d W(s), \quad t \in I_{j} .
$$

Note that $R_{1}^{H}, \ldots, R_{m}^{H}$ are independent processes satisfying

$$
\left\|R_{j}^{H}\right\|_{L_{q}\left(I_{j}\right)} \stackrel{d}{=}\left|I_{j}\right|^{H+1 / q}\left\|R^{H}\right\|_{L_{q}[0,1]}
$$

with $R^{H}$ given by (4.1). Hence, from (4.2) and (4.12) we derive

$$
\lim _{\varepsilon \rightarrow 0} \varepsilon^{1 / H} \log \mathbb{P}\left(\left\|\rho_{j} R_{j}^{H}\right\|_{L_{q}\left(I_{j}\right)} \leq \varepsilon\right)=-c(H, q) \cdot \rho_{j}^{1 / H} \cdot\left|I_{j}\right|^{1+1 / H q}
$$

for $1 \leq j \leq m$.

Next, we use the following summation rule for the small ball asymptotic (see e.g. [10] or Corollary 3.1 in [13]). It asserts that if for each member of a finite family $\left(\zeta_{j}\right)_{j=1}^{m}$ of non-negative independent random variables one has

$$
\lim _{\varepsilon \rightarrow 0} \varepsilon^{\alpha} \log \mathbb{P}\left(\zeta_{j} \leq \varepsilon\right)=-b_{j},
$$

then

$$
\lim _{\varepsilon \rightarrow 0} \varepsilon^{\alpha} \log \mathbb{P}\left(\sum_{j=1}^{m} \zeta_{j} \leq \varepsilon\right)=-\left(\sum_{j=1}^{m} b_{j}^{\frac{1}{1+\alpha}}\right)^{1+\alpha} .
$$

We apply this rule with $\alpha=1 / H q$ and $\zeta_{j}=\left\|\rho_{j} R_{j}^{H}\right\|_{L_{q}\left(I_{j}\right)}^{q}$ and obtain by 4.13)

$$
\begin{aligned}
& \lim _{\varepsilon \rightarrow 0} \varepsilon^{1 / H} \log \mathbb{P}\left(\left\|\sum_{j=1}^{m} \rho_{j} R_{j}^{H}\right\|_{L_{q}(I)} \leq \varepsilon\right) \\
& =-c(H, q) \cdot\left(\sum_{j=1}^{m} \rho_{j}^{\frac{1}{H+1 / q}}\left|I_{j}\right|\right)^{1+1 / H q}=-c(H, q) \cdot\|\rho\|_{L_{r}(I)}^{1 / H} .
\end{aligned}
$$


From Lemma 4.2 and (4.10) one derives for each $\eta \in(0,1)$ the following inequality:

$$
\begin{aligned}
& \mathbb{P}\left(\left\|\rho B^{H}\right\|_{L_{q}(I)} \leq \varepsilon\right) \\
& \quad \geq \mathbb{P}\left(\left\|\rho Z^{H}\right\|_{L_{q}(I)} \leq \varepsilon / K(\eta)\right) \cdot \mathbb{P}\left(\left\|\sum_{j=1}^{m} \rho_{j} R_{j}^{H}\right\|_{L_{q}(I)} \leq(1-\eta) \varepsilon\right) .
\end{aligned}
$$

In view of Lemma 4.3 this leads to

$$
\begin{aligned}
& \liminf _{\varepsilon \rightarrow 0} \varepsilon^{1 / H} \log \mathbb{P}\left(\left\|\rho B^{H}\right\|_{L_{q}(I)} \leq \varepsilon\right) \\
& \geq \liminf _{\varepsilon \rightarrow 0} \varepsilon^{1 / H} \log \mathbb{P}\left(\left\|\sum_{j=1}^{m} \rho_{j} R_{j}^{H}\right\|_{L_{q}(I)} \leq(1-\eta) \varepsilon\right) .
\end{aligned}
$$

Combining (4.15) with (4.14) and taking the limit $\eta \rightarrow 0$ in the right-hand side completes the proof for interval step functions $\rho$.

Now let $\rho \in L_{q}(I)$ be arbitrary. For $\delta>0$ we find (cf. Lemma 4.4 in [16]) an interval step function $\psi$ such that

$$
\|\bar{\rho}\|_{L_{q}(I)}<\delta \quad \text { and } \quad\|\psi\|_{L_{r}(I)}=\|\rho\|_{L_{r}(I)},
$$

where $\bar{\rho}:=[\rho-\psi]_{+}$. We have $\rho \leq \psi+\bar{\rho}$, hence another application of Lemma 4.2 yields

$$
\begin{aligned}
& \mathbb{P}\left(\left\|\rho B^{H}\right\|_{L_{q}(I)} \leq \varepsilon\right) \geq \mathbb{P}\left(\left\|\psi B^{H}+\bar{\rho} B^{H}\right\|_{L_{q}(I)} \leq \varepsilon\right) \\
& \quad \geq \mathbb{P}\left(\left\|\psi B^{H}\right\|_{L_{q}(I)} \leq(1-\eta) \varepsilon\right) \cdot \mathbb{P}\left(\left\|\bar{\rho} B^{H}\right\|_{L_{q}(I)} \leq \varepsilon / K(\eta)\right) .
\end{aligned}
$$

Since by 4.16)

$$
\left\|\bar{\rho} B^{H}\right\|_{L_{q}(I)} \leq\|\bar{\rho}\|_{L_{q}(I)} \cdot\left\|B^{H}\right\|_{L_{\infty}(I)} \leq \delta \cdot\left\|B^{H}\right\|_{L_{\infty}(I)}
$$

from (4.3) we derive

$$
\liminf _{\varepsilon \rightarrow 0} \varepsilon^{1 / H} \log \mathbb{P}\left(\left\|\bar{\rho} B^{H}\right\|_{L_{q}(I)} \leq \varepsilon / K(\eta)\right) \geq-c(H, \infty) \cdot(K(\eta) \cdot \delta)^{1 / H} .
$$

On the other hand (recall that $\psi$ is an interval step function), by the first step and by (4.16) it follows that

$$
\liminf _{\varepsilon \rightarrow 0} \varepsilon^{1 / H} \log \mathbb{P}\left(\left\|\psi B^{H}\right\|_{L_{q}(I)} \leq(1-\eta) \varepsilon\right)
$$

$$
\geq-c(H, q) \cdot\|\psi\|_{L_{r}(I)}^{1 / H} \cdot(1-\eta)^{1 / H}=-c(H, q) \cdot\|\rho\|_{L_{r}(I)}^{1 / H} \cdot(1-\eta)^{1 / H} .
$$

Combining (4.17), (4.18) and (4.19) finally leads to

$$
\begin{aligned}
& \liminf _{\varepsilon \rightarrow 0} \varepsilon^{1 / H} \log \mathbb{P}\left(\left\|\rho B^{H}\right\|_{L_{q}(I)} \leq \varepsilon\right) \\
& \geq-c(H, q) \cdot\|\rho\|_{L_{r}(I)}^{1 / H} \cdot(1-\eta)^{1 / H}-c(H, \infty) \cdot(K(\eta) \cdot \delta)^{1 / H},
\end{aligned}
$$

hence, if we first take the limit $\delta \rightarrow 0$ and then $\eta \rightarrow 0$, we obtain (4.8).

Theorem 4.5. For any weight function $\rho$ on $[0, \infty)$ and any $q \in[1, \infty)$ one has

$$
\limsup _{\varepsilon \rightarrow 0} \varepsilon^{1 / H} \log \mathbb{P}\left(\left\|\rho R^{H}\right\|_{L_{q}(0, \infty)} \leq \varepsilon\right) \leq-c(H, q) \cdot\|\rho\|_{L_{r}(0, \infty)}^{1 / H}
$$

with $r>0$ defined by [1.4). 
Proof. Again we start with proving (4.20) for interval step functions. Thus suppose $\rho$ may be written as $\rho=\sum_{j=1}^{m} \rho_{j} \mathbf{1}_{I_{j}}$ with $\rho_{j}$ 's, $I$ and $I_{j}$ 's as in (4.9). We now decompose

$$
\rho R^{H}=\rho \tilde{Z}^{H}+\sum_{j=1}^{m} \rho_{j} R_{j}^{H}
$$

with $R_{j}^{H}$ 's defined by (4.11) and with

$$
\tilde{Z}^{H}(t):=\int_{0}^{s_{j}}(t-s)^{H-1 / 2} d W(s), \quad t \in I_{j} .
$$

By Anderson's inequality

$$
\mathbb{P}\left(\left\|\rho Z^{H}\right\|_{L_{q}(I)} \leq \varepsilon\right) \leq \mathbb{P}\left(\left\|\rho \tilde{Z}^{H}\right\|_{L_{q}(I)} \leq \varepsilon\right)
$$

for $Z^{H}$ defined by (4.5), hence Lemma 4.3 lets us conclude that

$$
\lim _{\varepsilon \rightarrow 0} \varepsilon^{1 / H} \log \mathbb{P}\left(\left\|\rho \tilde{Z}^{H}\right\|_{L_{q}(I)} \leq \varepsilon\right)=0 .
$$

On the other hand, by (4.14) we have

$$
\lim _{\varepsilon \rightarrow 0} \varepsilon^{1 / H} \log \mathbb{P}\left(\left\|\sum_{j=1}^{m} \rho_{j} R_{j}^{H}\right\|_{L_{q}(I)} \leq \varepsilon\right)=-c(H, q) \cdot\|\rho\|_{L_{r}(I)}^{1 / H} .
$$

Next we apply Lemma 4.2 to (4.21) and obtain

$$
\begin{aligned}
-c(H, q) \cdot\|\rho\|_{L_{r}(I)}^{1 / H} & =\lim _{\varepsilon \rightarrow 0} \varepsilon^{1 / H} \log \mathbb{P}\left(\left\|\sum_{j=1}^{m} \rho_{j} R_{j}^{H}\right\|_{L_{q}(I)} \leq \varepsilon\right) \\
& \geq \limsup _{\varepsilon \rightarrow 0} \varepsilon^{1 / H} \log \mathbb{P}\left(\left\|\rho R^{H}\right\|_{L_{q}(I)} \leq(1-\eta) \varepsilon\right) \\
& +\liminf _{\varepsilon \rightarrow 0} \varepsilon^{1 / H} \log \mathbb{P}\left(\left\|\rho \tilde{Z}^{H}\right\|_{L_{q}(I)} \leq \varepsilon / K(\eta)\right)
\end{aligned}
$$

which by (4.22) implies

$$
\limsup _{\varepsilon \rightarrow 0} \varepsilon^{1 / H} \log \mathbb{P}\left(\left\|\rho R^{H}\right\|_{L_{q}(I)} \leq \varepsilon\right) \leq-c(H, q) \cdot(1-\eta)^{1 / H} \cdot\|\rho\|_{L_{r}(I)}^{1 / H} .
$$

Thus (4.20) follows by taking $\eta \rightarrow 0$.

Let us now treat the general case. First note that it suffices to prove (4.20) for bounded $\rho$ 's supported on a bounded closed interval $I \subset(0, \infty)$. Since $I$ is strictly separated from the critical points 0 and $\infty$, for a given $\delta>0$ we find a (non-negative) interval step function $\psi$ on $I$ satisfying

$$
\|\psi-\rho\|_{L_{r}(I)}<\delta
$$

as well as

$$
\left|\|\rho\|_{L_{r}(I)}^{1 / H}-\|\psi\|_{L_{r}(I)}^{1 / H}\right|<\delta .
$$


Because of $\psi \leq \rho+[\psi-\rho]_{+}$from Lemma4.2] and the first step we derive

$$
\begin{aligned}
-c(H, q) \cdot\|\psi\|_{L_{r}(I)}^{1 / H} & \geq \limsup _{\varepsilon \rightarrow 0} \varepsilon^{1 / H} \log \mathbb{P}\left(\left\|\psi R^{H}\right\|_{L_{q}(I)} \leq \varepsilon\right) \\
& \geq \limsup _{\varepsilon \rightarrow 0} \varepsilon^{1 / H} \log \mathbb{P}\left(\left\|\rho R^{H}\right\|_{L_{q}(I)} \leq(1-\eta) \varepsilon\right) \\
& +\liminf _{\varepsilon \rightarrow 0} \varepsilon^{1 / H} \log \mathbb{P}\left(\left\|[\psi-\rho]_{+} R^{H}\right\|_{L_{q}(I)} \leq \varepsilon / K(\eta)\right) .
\end{aligned}
$$

The last term in 4.25) can be estimated by Theorem 4.4, hence by (4.23) and (4.24) it follows that

$$
\begin{aligned}
& \limsup _{\varepsilon \rightarrow 0} \varepsilon^{1 / H} \log \mathbb{P}\left(\left\|\rho R^{H}\right\|_{L_{q}(I)} \leq \varepsilon\right) \\
& \quad \leq(1-\eta)^{1 / H}\left\{-c(H, q) \cdot\|\psi\|_{L_{r}(I)}^{1 / H}+c(H, q) \cdot K(\eta)^{1 / H} \cdot\|\psi-\rho\|_{r}^{1 / H}\right\} \\
& \leq(1-\eta)^{1 / H}\left\{-c(H, q) \cdot\left(\|\rho\|_{L_{r}(I)}^{1 / H}-\delta\right)+c(H, q) \cdot K(\eta)^{1 / H} \cdot \delta^{1 / H}\right\} .
\end{aligned}
$$

Finally, first taking $\delta \rightarrow 0$ and then $\eta \rightarrow 0$ proves (4.20) for arbitrary $\rho$ 's.

Combining the previous results we obtain the following.

Theorem 4.6. Let $\rho$ be a weight function on $(0, \infty)$ such that for some $q \in[0, \infty)$ we have $|\rho|_{r}<\infty$. Recall that $1 / r=H+1 / q$. Then

$$
\begin{aligned}
\lim _{\varepsilon \rightarrow 0} \varepsilon^{1 / H} \log \mathbb{P}\left(\left\|\rho R^{H}\right\|_{L_{q}(0, \infty)} \leq \varepsilon\right) & =\lim _{\varepsilon \rightarrow 0} \varepsilon^{1 / H} \log \mathbb{P}\left(\left\|\rho B^{H}\right\|_{L_{q}(0, \infty)} \leq \varepsilon\right) \\
& =-c(H, q) \cdot\|\rho\|_{L_{r}(0, \infty)}^{1 / H} .
\end{aligned}
$$

Proof. Since $|\rho|_{r}<\infty$, for a given $\delta>0$ we may split $\rho$ into the sum of two nonnegative functions $\rho^{(1)}$ and $\rho^{(2)}$ such that $\rho^{(1)}$ is supported on a closed bounded interval in $(0, \infty)$ and that $\left|\rho^{(2)}\right|_{r}<\delta$. Hence Theorem 4.4 applies to $\rho^{(1)}$ and gives

$$
\liminf _{\varepsilon \rightarrow 0} \varepsilon^{1 / H} \log \mathbb{P}\left(\left\|\rho^{(1)} B^{H}\right\|_{L_{q}(0, \infty)} \leq \varepsilon\right) \geq-c(H, q) \cdot\|\rho\|_{L_{r}(0, \infty)}^{1 / H}
$$

while by Theorem 4.1 we have

$$
\liminf _{\varepsilon \rightarrow 0} \varepsilon^{1 / H} \log \mathbb{P}\left(\left\|\rho^{(2)} B^{H}\right\|_{L_{q}(0, \infty)} \leq \varepsilon\right) \geq-c \cdot\left|\rho^{(2)}\right|_{r}^{1 / H} \geq-c \cdot \delta^{1 / H} .
$$

Applying Lemma 4.2, for each $\eta \in(0,1)$ it follows that

$$
\begin{aligned}
& \liminf _{\varepsilon \rightarrow 0} \varepsilon^{1 / H} \log \mathbb{P}\left(\left\|\rho B^{H}\right\|_{L_{q}(0, \infty)} \leq \varepsilon\right) \\
& \geq \quad-c(H, q) \cdot\|\rho\|_{L_{r}(0, \infty)}^{1 / H} \cdot(1-\eta)^{-1 / H}-c \cdot K(\eta)^{1 / H} \cdot \delta^{1 / H} .
\end{aligned}
$$

Taking $\delta \rightarrow 0$ and then $\eta \rightarrow 0$ finally implies that

$$
\liminf _{\varepsilon \rightarrow 0} \varepsilon^{1 / H} \log \mathbb{P}\left(\left\|\rho B^{H}\right\|_{L_{q}(0, \infty)} \leq \varepsilon\right) \geq-c(H, q) \cdot\|\rho\|_{L_{r}(0, \infty)}^{1 / H} .
$$

By Anderson's inequality, the same is true for $R^{H}$.

On the other hand, Anderson's inequality and Theorem 4.5 let us conclude that

$$
\begin{aligned}
& \limsup _{\varepsilon \rightarrow 0} \varepsilon^{1 / H} \log \mathbb{P}\left(\left\|\rho B^{H}\right\|_{L_{q}(0, \infty)} \leq \varepsilon\right) \\
& \quad \leq \limsup _{\varepsilon \rightarrow 0} \varepsilon^{1 / H} \log \mathbb{P}\left(\left\|\rho R^{H}\right\|_{L_{q}(0, \infty)} \leq \varepsilon\right) \leq-c(H, q) \cdot\|\rho\|_{L_{r}(0, \infty)}^{1 / H}
\end{aligned}
$$

completing the proof. 
Some comments to the case $q=\infty$ which is not covered by Theorem 4.4, Theorem 4.5 and by Theorem 4.6 above. The results are also valid in this case with $\rho$ replaced by its regularization $\rho^{*}$ defined by

$$
\rho^{*}(s):=\lim _{\delta \rightarrow 0} \operatorname{ess}_{\{x:|x-s|<\delta\}} \rho(s) .
$$

The proofs practically do not change. We only have to use Lemma 4.5 in [16] instead of Lemma 4.4 to approximate the weight function by interval step functions. For more details we refer to [16].

\section{Average Kolmogorov numbers and their application}

The proof of Theorem 2.1 relies upon properties of so-called average Kolmogorov numbers of operators. For a separable Hilbert space $\mathcal{H}$ we denote by $\mathcal{G}(\mathcal{H}, E)$ the set of operators $T$ from $\mathcal{H}$ into $E$ such that the series

$$
X_{T}:=\sum_{k=1}^{\infty} \xi_{k} T f_{k}
$$

converges a.s. in $E$ for some (each) ONB $\left(f_{k}\right)_{k \geq 1}$ in $\mathcal{H}$. Here $\left(\xi_{k}\right)_{k \geq 1}$ is an i.i.d. sequence of $\mathcal{N}(0,1)$-distributed random variables. Recall that (with suitable $T \in$ $\mathcal{G}(\mathcal{H}, E))$ any centered Gaussian $E$-valued vector may be represented in the form (5.1).

For $T \in \mathcal{G}(\mathcal{H}, E)$ we may now define its $n$-th average Kolmogorov number by

$$
g_{n}(T):=g_{n}\left(X_{T}\right)=\inf \left\{\left(\mathbb{E}\left\|Q_{N} X_{T}\right\|^{2}\right)^{1 / 2}: \operatorname{dim} N<n\right\}
$$

with $X_{T}$ defined by (5.1). In particular, $l(T):=g_{1}(T)=\left(\mathbb{E}\left\|X_{T}\right\|^{2}\right)^{1 / 2}$ is the wellknown $l$-norm of $T$ and $[\mathcal{G}(\mathcal{H}, E), l]$ is a Banach space (cf. [22] for more details).

The following properties of $g_{n}(T)$ are either straightforward or may be found in 6]. For the definition of the "ordinary" Kolmogorov numbers $d_{n}(T)$, we refer to [21].

(a) For $q \in[1, \infty)$ set

$$
g_{n}^{(q)}(T):=\inf \left\{\left(\mathbb{E} \inf _{y \in N}\left\|X_{T}-y\right\|^{q}\right)^{1 / q}: \operatorname{dim}(N)<n\right\} .
$$

Then there exist two constants $c_{1}, c_{2}>0$ depending only on $q$ such that

$$
c_{1} g_{n}(T) \leq g_{n}^{(q)}(T) \leq c_{2} g_{n}(T) .
$$

(b) By a deep result in 20 there exists a universal $c>0$ such that for all $T \in \mathcal{G}(\mathcal{H}, E)$

$$
n^{1 / 2} \cdot d_{2 n-1}(T) \leq c \cdot g_{n}(T) .
$$

Consequently, by Carl's inequality

$$
\sup _{n \geq 1} n^{1 / \beta+1 / 2} e_{n}(T) \leq c(\beta) \cdot \sup _{n \geq 1} n^{1 / \beta+1 / 2} d_{n}(T)
$$

(see e.g. 22] or [4] for a proof) it follows that

$$
\sup _{n \geq 1} n^{1 / \beta+1 / 2} e_{n}(T) \leq c \cdot \sup _{n \geq 1} n^{1 / \beta} g_{n}(T)
$$

for any $\beta>0$ and with $c>0$ depending only on $\beta$. 
(c) The following is a slightly improved version of a result due to G. Pisier (see [22], page 141). If $T^{*}: E^{*} \rightarrow \mathcal{H}$ denotes the dual of $T \in \mathcal{G}(\mathcal{H}, E)$, then for some $c_{1}, c_{2}>0$ we have

$$
g_{n}(T) \leq c_{1} \cdot \sum_{k \geq c_{2} n} k^{-1 / 2} e_{k}\left(T^{*}\right) .
$$

By combining this bound with the estimates from [25], we obtain

$$
\sup _{n \geq 1} n^{1 / \beta} g_{n}(T) \leq c \cdot \sup _{n \geq 1} n^{1 / \beta+1 / 2} e_{n}(T)
$$

for any $\beta>0$.

The main advantage of the numbers $g_{n}$ (in contrast to the entropy numbers) is that they are well related to the $l_{q}$-summation of operators, as we shall see in a moment. Before doing so, let us first state conditions ensuring $T^{q} \in \mathcal{G}\left(\mathcal{H}, E^{q}\right)$ provided each operator $T_{j}$ belongs to $\mathcal{G}\left(\mathcal{H}, E_{j}\right)$.

Theorem 5.1. If the operators $T_{j} \in \mathcal{G}\left(\mathcal{H}, E_{j}\right)$ satisfy

$$
\sum_{j=1}^{\infty} l\left(T_{j}\right)^{q}<\infty
$$

then their $l_{q}$-sum $T^{q}$ belongs to $\mathcal{G}\left(\mathcal{H}, E^{q}\right)$ and

$$
l\left(T^{q}\right) \leq c_{q} \cdot\left[\sum_{j=1}^{\infty} l\left(T_{j}\right)^{q}\right]^{1 / q} .
$$

Proof. For $m \geq 0$ let $P_{m}: E^{q} \rightarrow E^{q}$ be defined by

$$
P_{m}(x):=\left(x_{1}, \ldots, x_{m}, 0,0, \ldots\right), \quad x=\left(x_{j}\right)_{j \geq 1} .
$$

Of course, we have $P_{m} \circ T^{q} \in \mathcal{G}\left(\mathcal{H}, E^{q}\right)$ and, if $n>m$, then by the equivalence of all moments of Gaussian vectors it follows that

$$
l\left(P_{n} \circ T^{q}-P_{m} \circ T^{q}\right) \leq c_{q}\left[\sum_{j=m+1}^{n} l\left(T_{j}\right)^{q}\right]^{1 / q} .
$$

Hence, by (5.4) and the completeness of $\left[\mathcal{G}\left(\mathcal{H}, E^{q}\right), l\right]$ we get $T^{q} \in \mathcal{G}\left(\mathcal{H}, E^{q}\right)$ as asserted.

From (5.6) we derive

$$
l\left(P_{n} \circ T^{q}\right) \leq c_{q}\left[\sum_{j=1}^{n} l\left(T_{j}\right)^{q}\right]^{1 / q}
$$

which, of course, implies (5.5) by taking the limit $n \rightarrow \infty$.

The next result is the key for the proof of Theorem 2.1.

Theorem 5.2. Let $\beta>0$ be given and suppose we have

$$
\sup _{n \geq 1} n^{1 / \beta} g_{n}\left(T_{j}\right):=\kappa_{j}<\infty .
$$


Then

$$
\sup _{n \geq 1} n^{1 / \beta} g_{n}\left(T^{q}\right) \leq c_{q} \cdot\left(\sum_{j=1}^{\infty} \kappa_{j}^{r}\right)^{1 / r}
$$

with $1 / r=1 / \beta+1 / q$ and $c_{q}$ depending only on $q$.

Proof. Of course, we may assume $\sum \kappa_{j}^{r}<\infty$, hence $\sum \kappa_{j}^{q}<\infty$ as well. Consequently,

$$
\sum_{j=1}^{\infty} l\left(T_{j}\right)^{q}=\sum_{j=1}^{\infty} g_{1}\left(T_{j}\right)^{q} \leq \sum_{j=1}^{\infty} \kappa_{j}^{q}<\infty
$$

which implies $T^{q} \in \mathcal{G}\left(\mathcal{H}, E^{q}\right)$ by Theorem 5.1.

Let $n_{j} \geq 1$ be any sequence of integers with $m:=\sum_{j=1}^{\infty}\left(n_{j}-1\right)<\infty$. For this sequence, we choose subspaces $N_{j} \subseteq E_{j}$ satisfying $\operatorname{dim}\left(N_{j}\right) \leq n_{j}-1$ and

$$
\mathbb{E}\left\|Q_{N_{j}} X_{T_{j}}\right\|^{q} \leq 2^{q} g_{n_{j}}^{(q)}\left(T_{j}\right)^{q} .
$$

Next we define $N \subseteq E^{q}$ by

$$
N:=\left\{x=\left(x_{j}\right)_{j \geq 1}: x_{j} \in N_{j}\right\}
$$

and observe that $\operatorname{dim}(N) \leq m$. Hence, by property (a) and (5.7),

$$
\begin{aligned}
g_{m+1}^{(q)}\left(T^{q}\right)^{q} & \leq \mathbb{E}\left\|Q_{N} X_{T^{q}}\right\|^{q}=\sum_{j=1}^{\infty} \mathbb{E}\left\|Q_{N_{j}} X_{T_{j}}\right\|^{q} \leq 2^{q} \sum_{j=1}^{\infty} g_{n_{j}}^{(q)}\left(T_{j}\right)^{q} \\
& \leq c^{q} \sum_{j=1}^{\infty} g_{n_{j}}\left(T_{j}\right)^{q} \leq c^{q} \sum_{j=1}^{\infty} \kappa_{j}^{q} \cdot n_{j}^{-q / \beta} .
\end{aligned}
$$

For a fixed natural number $n$ we now choose integers $n_{j}$ satisfying

$$
n_{j}-1<n \cdot \frac{\kappa_{j}^{r}}{\sum_{i=1}^{\infty} \kappa_{i}^{r}} \leq n_{j}
$$

for all $j \geq 1$. Of course, only a finite number of the $n_{j}$ 's can be different from 1 , thus $m:=\sum_{j=1}^{\infty}\left(n_{j}-1\right)<\infty$ and by (5.9) it follows that $m<n$. From this, (5.8) and (5.9) we derive

$$
\begin{aligned}
g_{n}^{(q)}\left(T^{q}\right) & \leq g_{m+1}^{(q)}\left(T^{q}\right) \leq c \cdot n^{-1 / \beta}\left(\sum_{j=1}^{\infty} \kappa_{j}^{q-q r / \beta}\right)^{1 / q} \cdot\left(\sum_{i=1}^{\infty} \kappa_{i}^{r}\right)^{1 / \beta} \\
& =c \cdot n^{-1 / \beta}\left(\sum_{j=1}^{\infty} \kappa_{j}^{r}\right)^{1 / r}
\end{aligned}
$$

by the choice of $r$. Combining this with property (a) of the $g_{n}$ 's completes the proof.

Proof of Theorem 2.1. Let $\beta>0$ be defined by $1 / \beta:=1 / \gamma-1 / 2$. In view of (5.3) assumption (2.1) leads to $\sup _{n \geq 1} n^{1 / \beta} g_{n}\left(T_{j}\right) \leq c_{1}(\beta) \cdot \kappa_{j}$ for all $j=1,2, \ldots$. Hence, Theorem 5.2 yields

$$
\sup _{n \geq 1} n^{1 / \beta} g_{n}\left(T^{q}\right) \leq c_{2}(q, \beta) \cdot\left(\sum_{j=1}^{\infty} \kappa_{j}^{r}\right)^{1 / r}
$$


where $1 / r=1 / \beta+1 / q=1 / \gamma+1 / q-1 / 2$. Finally, from (5.2) and (5.10) we derive

$$
\sup _{n \geq 1} n^{1 / \gamma} e_{n}\left(T^{q}\right)=\sup _{n \geq 1} n^{1 / \beta+1 / 2} e_{n}\left(T^{q}\right) \leq c_{3}(q, \beta) \cdot\left(\sum_{j=1}^{\infty} \kappa_{j}^{r}\right)^{1 / r}
$$

as asserted.

Problem. Suppose that the operators $T_{j}$ are defined on some $L_{p}$-space with $1<$ $p<\infty$ and satisfy (2.1). What is the largest possible $r>0$ (depending on $\gamma, q$ and $p$ ) for which (2.2) holds? Which restrictions on the parameter $\gamma$ are necessary?

\section{BundLes of METRIC SPACES}

For $j \in \mathbb{N}$ let $\left(K_{j}, d_{j}\right)$ be compact metric spaces and suppose that in each space $K_{j}$ a special point $t_{j}^{*}$ is marked. Then we define the bundle space $\left(K_{\infty}, d_{\infty}\right)$ as a (disjoint) union of the $K_{j}$ 's where all $t_{j}^{*}$ 's are identified with one point $t^{*} \in K_{\infty}$. The metric $d_{\infty}$ on $K_{\infty}$ is then given by

$$
d_{\infty}\left(t_{1}, t_{2}\right):= \begin{cases}d_{j}\left(t_{1}, t_{2}\right) & \text { if } t_{1}, t_{2} \in K_{j}, \\ d_{j_{1}}\left(t_{1}, t_{j_{1}}^{*}\right)+d_{j_{2}}\left(t_{2}, t_{j_{2}}^{*}\right) & \text { if } t_{1} \in K_{j_{1}}, t_{2} \in K_{j_{2}}, j_{1} \neq j_{2} .\end{cases}
$$

Observe that $\left(K_{\infty}, d_{\infty}\right)$ is not necessarily compact. It is so (as can easily be seen) iff we have $\operatorname{diam}\left(K_{j}\right) \rightarrow 0$ as $j \rightarrow \infty$. Assuming this, it is natural to ask how certain compactness properties of the $K_{j}$ 's carry over to those of the bundle space $K_{\infty}$. The next theorem answers this question.

Theorem 6.1. Suppose the family of metric spaces $\left(K_{j}, d_{j}\right)$ generates the bundle space $\left(K_{\infty}, d_{\infty}\right)$ and assume that for some $\sigma>0$ and for any $j \geq 1$ there exist $\rho_{j}>0$ such that

$$
\varepsilon_{n}\left(K_{j}, d_{j}\right)<\rho_{j} \cdot n^{-1 / \sigma}, \quad n \in \mathbb{N}
$$

Then

$$
\varepsilon_{n}\left(K_{\infty}, d_{\infty}\right) \leq 2^{2 / \sigma+1} \tilde{\rho} \cdot n^{-1 / \sigma}, \quad n \in \mathbb{N}
$$

with $\tilde{\rho}=\left(\sum_{j=1}^{\infty} \rho_{j}^{\sigma}\right)^{1 / \sigma}$.

We omit the elementary and relatively simple proof of Theorem6.1.

Before proceeding further we need certain compactness properties of Hölder operators with values in a space of continuous functions. More precisely, the following may be found in [5], Sect. 5.10.

Theorem 6.2. Let $\mathcal{H}$ be a Hilbert space and let $\rho_{0}, \sigma>0$ and $\alpha \in(0,1]$. Then there exists a constant $c=c(\sigma, \alpha)>0$ such that the following is valid: If $(K, d)$ is a compact space with

$$
\varepsilon_{n}(K, d) \leq \rho_{0} \cdot n^{-1 / \sigma}, \quad n \in \mathbb{N},
$$

then for each $\alpha$-Hölder operator $T: \mathcal{H} \rightarrow C(K, d)$ we have

$$
e_{n}(T) \leq c \cdot \rho_{0}^{\alpha} \cdot\|T\|_{\alpha} \cdot n^{-1 / 2-\alpha / \sigma}, \quad n \in \mathbb{N} .
$$


If $C_{0}\left(K_{j}, d_{j}\right)$ denotes the set of $f \in C\left(K_{j}, d_{j}\right)$ with $f\left(t_{j}^{*}\right)=0$, the $l_{\infty}$-sum of the $C_{0}\left(K_{j}, d_{j}\right)$ 's may obviously be viewed as $\tilde{C}_{0}\left(K_{\infty}, d_{\infty}\right)$, the space of functions defined on $K_{\infty}$, continuous on every $K_{j}$, and vanishing at $t^{*}$. Note that such functions need not be continuous on the entire space $K_{\infty}$. Suppose now we are given a family $T_{j}, j \in \mathbb{N}$, of operators, mapping a Hilbert space $\mathcal{H}$ into $C\left(K_{j}, d_{j}\right)$. Our aim is to paste them together to a single operator $T^{\infty}$. To do so, we have to assume that each operator $T_{j}$ maps even into $C_{0}\left(K_{j}, d_{j}\right)$, i.e. if $t_{j}^{*} \in K_{j}$ are the marked points, then we suppose that for $j \in \mathbb{N}$ we have

$$
\left(T_{j} h\right)\left(t_{j}^{*}\right)=0, \quad h \in \mathcal{H} .
$$

Under this assumption $T^{\infty}: \mathcal{H} \rightarrow \tilde{C}_{0}\left(K_{\infty}, d_{\infty}\right)$ with

$$
\left(T^{\infty} h\right)(t):=\left(T_{j} h\right)(t) \quad \text { for } t \in K_{j}
$$

is well defined.

Now we are in a position to verify the following basic ingredient in the proof of Theorem 2.2,

Theorem 6.3. Let $T_{j}: \mathcal{H} \rightarrow C\left(K_{j}, d_{j}\right)$ be $\alpha$-Hölder operators for some $\alpha \in(0,1]$ satisfying (6.3) and suppose that for each $j, n \in \mathbb{N}$ we have

$$
\varepsilon_{n}\left(K_{j}, d_{j}\right)<\rho_{j} \cdot n^{-1 / \sigma}
$$

for some $\rho_{j}>0$ and $\sigma>0$. Then

$$
e_{n}\left(T^{\infty}\right) \leq c \cdot \tilde{\rho} \cdot n^{-1 / 2-\alpha / \sigma},
$$

where

$$
\tilde{\rho}:=\left(\sum_{j=1}^{\infty}\left\|T_{j}\right\|_{\alpha}^{\sigma / \alpha} \rho_{j}^{\sigma}\right)^{\alpha / \sigma} .
$$

Proof. First suppose that the $T_{j}$ 's are 1 -Hölder with $\left\|T_{j}\right\|_{1} \leq 1$. We claim that then $T^{\infty}$ is 1 -Hölder with $\left\|T^{\infty}\right\|_{1} \leq 1$. To verify this, take $h \in \mathcal{H}$ with $\|h\| \leq 1$. For $t$ and $t^{\prime}$ in the same $K_{j}$ it follows that

$$
\left|\left(T^{\infty} h\right)(t)-\left(T^{\infty} h\right)\left(t^{\prime}\right)\right|=\left|\left(T_{j} h\right)(t)-\left(T_{j} h\right)\left(t^{\prime}\right)\right| \leq d_{j}\left(t, t^{\prime}\right)=d_{\infty}\left(t, t^{\prime}\right) .
$$

On the other hand, if $t_{1} \in K_{j_{1}}$ and $t_{2} \in K_{j_{2}}$ with $j_{1} \neq j_{2}$, using (6.3) and (6.7) we get

$$
\begin{aligned}
& \left|\left(T^{\infty} h\right)\left(t_{1}\right)-\left(T^{\infty} h\right)\left(t_{2}\right)\right| \leq\left|\left(T_{j_{1}} h\right)\left(t_{1}\right)\right|+\left|\left(T_{j_{2}} h\right)\left(t_{2}\right)\right| \\
& \quad=\left|\left(T_{j_{1}} h\right)\left(t_{1}\right)-\left(T_{j_{1}} h\right)\left(t_{j_{1}}^{*}\right)\right|+\left|\left(T_{j_{2}} h\right)\left(t_{2}\right)-\left(T_{j_{2}} h\right)\left(t_{j_{2}}^{*}\right)\right| \\
& \quad \leq d_{j_{1}}\left(t_{1}, t_{j_{1}}^{*}\right)+d_{j_{2}}\left(t_{2}, t_{j_{2}}^{*}\right)=d_{\infty}\left(t_{1}, t_{2}\right)
\end{aligned}
$$

which proves our claim.

By Theorem6.1 we derive from (6.4) that

$$
\varepsilon_{n}\left(K_{\infty}, d_{\infty}\right) \leq c \cdot\left(\sum_{j=1}^{\infty} \rho_{j}^{\sigma}\right)^{1 / \sigma} \cdot n^{-1 / \sigma} .
$$

Since $T^{\infty}$ is 1 -Hölder, it maps even into $C\left(K_{\infty}, d_{\infty}\right)$, thus Theorem 6.2 applies and leads by (6.8) to

$$
e_{n}\left(T_{\infty}\right) \leq c \cdot\left(\sum_{j=1}^{\infty} \rho_{j}^{\sigma}\right)^{1 / \sigma} \cdot n^{-1 / 2-1 / \sigma} .
$$


The general case now follows easily. Indeed, for $\alpha$-Hölder operators $T_{j}$ we change the metric on $K_{j}$ to $\hat{d}_{j}\left(t, t^{\prime}\right):=d_{j}\left(t, t^{\prime}\right)^{\alpha} \cdot\left\|T_{j}\right\|_{\alpha}$. Then $T_{j}: \mathcal{H} \rightarrow C\left(K_{j}, \hat{d}_{j}\right)$ is 1-Hölder with $\left\|T_{j}\right\|_{1}=1$ and, furthermore,

$$
\varepsilon_{n}\left(K_{j}, \hat{d}_{j}\right)=\left\|T_{j}\right\|_{\alpha} \cdot \varepsilon_{n}\left(K_{j}, d_{j}\right)^{\alpha} \leq\left\|T_{j}\right\|_{\alpha} \cdot \rho_{j}^{\alpha} \cdot n^{-\alpha / \sigma} .
$$

If we now apply the result of the first step with $\tilde{\sigma}=\sigma / \alpha$ and $\tilde{\rho}_{j}=\left\|T_{j}\right\|_{\alpha} \cdot \rho_{j}^{\alpha}$, then we get (6.5) and (6.6) from (6.9), as asserted.

Proof of Theorem 2.2. Let us mark arbitrary points $t_{j}^{*} \in K_{j}$. With these points we introduce 1-dimensional operators $V_{j}: \mathcal{H} \rightarrow C\left(K_{j}, d_{j}\right)$ by $\left(V_{j} h\right)(t):=\left(T_{j} h\right)\left(t_{j}^{*}\right)$, $t \in K_{j}, h \in \mathcal{H}$. If $\tilde{T}_{j}:=T_{j}-V_{j}$, then the family $\left(\tilde{T}_{j}\right)_{j \geq 1}$ satisfies (6.3) and since $\left\|\tilde{T}_{j}\right\|_{\alpha}=\left\|T_{j}\right\|_{\alpha}$, by Theorem 6.3 we conclude that

$$
e_{n}\left(\tilde{T}^{\infty}\right) \leq c \cdot \tilde{\rho} \cdot n^{-1 / 2-\alpha / \sigma}
$$

with $\tilde{\rho}$ from (6.6).

Define as before $V^{\infty}: \mathcal{H} \rightarrow l_{\infty}\left(C\left(K_{j}, d_{j}\right): j \in \mathbb{N}\right)$ by $V^{\infty} h:=\left(V_{j} h\right)_{j \geq 1}, h \in$ $\mathcal{H}$. We have $T^{\infty}=\tilde{T}^{\infty}+V^{\infty}$, thus it remains to estimate $e_{n}\left(V^{\infty}\right)$. First note that we may regard $V^{\infty}$ as an operator from $\mathcal{H}$ into $l_{\infty}=l_{\infty}(\mathbb{N})$ with $V^{\infty} h=$ $\left(\left(T_{j} h\right)\left(t_{j}^{*}\right)\right)_{j \geq 1}, h \in \mathcal{H}$. Set

$$
\delta_{j}:=\rho_{j}^{\alpha} \cdot\left\|T_{j}\right\|_{\alpha}
$$

and define $S: \mathcal{H} \rightarrow l_{\infty}$ by

$$
S h:=\left(\delta_{j}^{-1} \cdot\left(T_{j} h\right)\left(t_{j}^{*}\right)\right)_{j \geq 1} .
$$

By Theorem 6.2 assumption (6.4) leads to

$$
e_{n}\left(T_{j}\right) \leq c \cdot \rho_{j}^{\alpha} \cdot\left\|T_{j}\right\|_{\alpha} n^{-\frac{1}{2}-\frac{\alpha}{\sigma}},
$$

which implies, in particular,

$$
\left\|T_{j}\right\|=e_{1}\left(T_{j}\right) \leq c \cdot \rho_{j}^{\alpha} \cdot\left\|T_{j}\right\|_{\alpha}=c \cdot \delta_{j} .
$$

Hence, by

$$
\|S\|=\sup _{\|h\| \leq 1} \sup _{j} \frac{\left(T_{j} h\right)\left(t_{j}^{*}\right)}{\delta_{j}} \leq \frac{\left\|T_{j}\right\|}{\delta_{j}} \leq c
$$

the operator $S$ defined by (6.12) is bounded from $\mathcal{H}$ into $l_{\infty}$. Let the diagonal operator $D: l_{\infty} \rightarrow l_{\infty}$ be generated by the $\delta_{j}$ 's from 6.11. Then, by the construction, we have $V^{\infty}=D \circ S$.

We will now apply (a special case of) a deep result due to B. Carl (cf. 33) which, when combined with an estimate of Tomczak-Jaegermann (cf. [25]), may be formulated in the following dual version.

Theorem 6.4. Let $T$ be an operator from a Hilbert space $\mathcal{H}$ into $l_{q}$ for some $q \in[1, \infty]$. If $T=D \circ S$ for some bounded operator $S: \mathcal{H} \rightarrow l_{\infty}$ and for a diagonal operator $D: l_{\infty} \rightarrow l_{q}$ generated by a sequence $\left(\delta_{j}\right)_{j \geq 1}$, then for $0<\gamma<2$ we have

$$
\sup _{n \geq 1} n^{1 / \gamma} e_{n}(T) \leq c \cdot\|S\| \cdot\left\|\left(\delta_{j}\right)_{j \geq 1}\right\|_{r, \infty} \leq c \cdot\|S\| \cdot\left(\sum_{j=1}^{\infty}\left|\delta_{j}\right|^{r}\right)^{1 / r},
$$

where $1 / r=1 / \gamma+1 / q-1 / 2$. 
Let us now apply Theorem 6.4 with $\delta_{j}$ from (6.11), $T=V^{\infty}$ and parameters $q=\infty, 1 / \gamma=1 / 2+\alpha / \sigma$, hence $1 / r=1 / \gamma-1 / 2=\alpha / \sigma$. Doing so, we get

$$
\sup _{n \geq 1} n^{1 / 2+\alpha / \sigma} e_{n}\left(V^{\infty}\right) \leq c \cdot\left(\sum_{j=1}^{\infty} \delta_{j}^{r}\right)^{1 / r}=c \cdot \tilde{\rho}
$$

and combining this with (6.10) completes the proof.

\section{ACKNOWLEDGEMENTS}

The authors are very grateful to A. Hinrichs (Jena) for drawing their attention to the important results in 3 .

\section{REFERENCES}

1. T. W. Anderson, The integral of symmetric unimodular functions over a symmetric convex set and some probability inequalities. Proc. Amer. Math. Soc. 6 (1955), 170-176. MR0069229 (16:1005a)

2. E. S. Belinsky and W. Linde, Small ball probabilities of fractional Brownian sheets via fractional integration operators. J. Theor. Probab. 15 (2002), 589-612. MR1922439(2004d:60092)

3. B. Carl, On a characterization of operators from $l_{q}$ into a Banach space of type $p$ with some applications to eigenvalue problems. J. Funct. Anal. 48 (1982), 394-407. MR0678178 (84i:47033)

4. B. Carl, I. Kyrezi and A. Pajor, Metric entropy of convex hulls in Banach spaces. J. London Math. Soc. 60 (1999), 871-896. MR.1753820 (2001c:46019)

5. B. Carl and I. Stephani, Entropy, Compactness and Approximation of Operators. Cambridge Univ. Press, Cambridge 1990. MR1098497 (92e:47002)

6. J. Creutzig, Relations between classical, average, and probabilistic Kolmogorov widths. Complexity 18 (2002), 287-303. MR1895087|(2002k:41041)

7. S. Gengembre, Petites déviations pour les processus fractionnaires. Memoire de DEA, Université Lille-I, 2002

8. J. Kuelbs and W. V. Li, Metric entropy and the small ball problem for Gaussian measures. J. Funct. Anal. 116 (1993), 133-157. MR.1237989 (94j:60078)

9. W. V. Li, A Gaussian correlation inequality and its applications to small ball probabilities. Electronic Commun. in Probability 12 (1999), 111-118. MR1741737 (2001j:60074)

10. W. V. Li, Small ball estimates for Gaussian Markov processes under $L_{p}-$ norm. Stoch. Proc. Appl. 92 (2001), 87-102. MR.1815180 (2001m:60090)

11. W. V. Li and W. Linde, Existence of small ball constants for fractional Brownian motions. C.R.A.S. Paris 326 (1998), Sér. I, 1329-1334. MR.1649147 (2000a:60077)

12. W. V. Li and W. Linde, Approximation, metric entropy and small ball estimates for Gaussian measures. Ann. Probab. 27 (1999), 1556-1578. MR,1733160 (2001c:60059)

13. W. V. Li and Q.-M. Shao, Gaussian processes: inequalities, small ball probabilities and applications. In: Shanbhag, D. N. (ed.) et al., Stochastic processes: Theory and methods. Amsterdam: North-Holland/Elsevier. Handb. Stat. 19 (2001), 533-597. MR1861734

14. M. A. Lifshits, Gaussian Random Functions Kluwer, Dordrecht, 1995. MR1472736 (98k:60059)

15. M. A. Lifshits, Asymptotic behavior of small ball probabilities. Probab. Theory and Math. Statist. Proc. VII International Vilnius Conference. Vilnius, VSP/TEV (1999), 453-468.

16. M. A. Lifshits and W. Linde, Approximation and Entropy Numbers of Volterra Operators with Application to Brownian Motion. Memoirs AMS 745 (2002), 1-87. MR.1895252 (2004g:47066)

17. M. A. Lifshits and T. Simon, Small ball probabilities for stable Riemann-Liouville processes. Preprint of the University of Evry. (2003) arXiv: math.PR/0305092

18. B. B. Mandelbrot and J. W. Van Ness, Fractional Brownian motions, fractional noises and applications. SIAM Review 10 (1968), 422-437. MR0242239 (39:3572)

19. D. Monrad and H. Rootzén, Small values of Gaussian processes and functional laws of the iterated logarithm. Probab. Th. Rel. Fields 101 (1995), 173-192. MR.1318191 (96a:60032) 
20. A. Pajor and N. Tomczak-Jaegermann, Subspaces of small codimension of finite dimensional Banach spaces. Proc. Amer. Math. Soc. 97 (1985), 637-642. MR0845980 (87i:46040)

21. A. Pietsch, Eigenvalues and s-Numbers. Cambridge Univ. Press, Cambridge 1987. MR0890520 (88j:47022b)

22. G. Pisier, The Volume of Convex Bodies and Banach Space Geometry. Cambridge Univ. Press, Cambridge 1989. MR1036275 (91d:52005)

23. G. Schechtman, T. Schlumprecht and J. Zinn, On the Gaussian measure of the intersection. Ann. Probab. 26 (1998), 346-357. MF,1617052 (99c:60032)

24. Q.-M. Shao, A note on small ball probability of Gaussian processes with stationary increments. J. Theoret. Probab. 6 (1993), 595-602. MR1230348 (95b:60041)

25. N. Tomczak-Jaegermann, Dualité des nombres d'entropie pour des opérateurs à valeurs dans un espace de Hilbert. C.R. Acad. Sci. Paris 305 (1987), 299-301. MR0910364 (89c:47027)

St. Petersburg State University, Postbox 104, 197372 St. Petersburg, Russia

E-mail address: lifts@mail.rcom.ru

Institut für Stochastik, Friedrich-Schiller-Universität Jena, Ernst-Abbe-Platz 2, 07743 Jena, Germany

E-mail address: lindew@minet.uni-jena.de 